\title{
Diffusive dynamics in an amorphous superionic conductor
}

\author{
Christoph Tietz $\odot,{ }^{*}$ Tobias Michael Fritz, Katharina Holzweber $\odot$, Michael Legenstein, and Bogdan Sepiol $\odot$ \\ University of Vienna, Faculty of Physics, Boltzmanngasse 5, 1090 Vienna, Austria \\ Michael Leitner $\odot^{\dagger}$ \\ Heinz Maier-Leibnitz, Zentrum (MLZ), Technische Universität München, Lichtenbergstraße 1, 85747 Garching, Germany
}

(Received 18 April 2020; revised 20 July 2020; accepted 5 October 2020; published 27 October 2020)

\begin{abstract}
The fast diffusion of alkali ions and resulting high ionic conductivity is a defining feature of alkali borate glasses. Here we report impedance spectroscopy and atomic-scale $\mathrm{x}$-ray photon correlation spectroscopy measurements on rubidium borate systems $\left(\mathrm{Rb}_{2} \mathrm{O}\right)_{x}\left(\mathrm{~B}_{2} \mathrm{O}_{3}\right)_{100-x}$ with particular focus on $x=30 \mathrm{~mol} \%$. We find that the coherently scattered intensity does not show temporal fluctuations on timescales corresponding to ionic diffusion. We conclude that the spatial configuration of alkali sites as stepping stones for ionic diffusion evolves on the same timescales as the borate backbone, and that the defect density is low, i.e., at a given instant, nearly all alkali sites are singly occupied.
\end{abstract}

DOI: 10.1103/PhysRevResearch.2.043141

\section{INTRODUCTION}

The study of diffusion in crystalline matter has been a fruitful area of activity for solid-state physics in the past decades. It has yielded yet another example where crystalline point defects, even though being present only in minute concentrations under typical circumstances, can be of foremost importance for describing the behavior of a material, just as with F centers and dopant impurities in insulators and semiconductors. In particular, diffusion in typical close-packed metals today is accepted to be the effect of the random walk of thermally created vacancies [1].

The situation is different for amorphous solid matter. An independent uncorrelated random walk of the atoms as in a gas, on the one hand, or vacancy-driven diffusion dynamics like in a crystal, on the other hand, constitute the extremal points of view, but with the absence of crystalline order any intermediate degree is a priori also possible and has been discussed [2-5]. A compounding factor here is that the transition from an ordinary liquid to a glass as an amorphous solid happens only gradually, necessitating low temperatures to study the peculiarities of the glassy state, so the classical experimental methods sensitive to the atomic scale of diffusion like quasielastic neutron or Mößbauer scattering requiring fast dynamics cannot be applied. As a consequence, direct investigations of diffusion have been done only by way of

\footnotetext{
*christoph.tietz@univie.ac.at

†michael.leitner@frm2.tum.de
}

Published by the American Physical Society under the terms of the Creative Commons Attribution 4.0 International license. Further distribution of this work must maintain attribution to the author(s) and the published article's title, journal citation, and DOI. simulations, while experimental evidence typically exists just for indirect quantities such as activation energies of diffusion.

The case of superionic conductors displays yet additional complications. The prototypical crystalline representative $\alpha$ $\mathrm{AgI}$ is commonly rationalized by the concept of the molten sublattice, which implies that the iodine anions make up a quasi-static bcc sublattice, while the small silver cations are dispersed over the bcc lattice' interstitial sites. At a given instant in time, only one in six of such sites is occupied, which together with the significant short-range order makes the $\mathrm{Ag}^{+}$ ions behave like a liquid [6].

Amongst the glassy superionic conductors, it is commonly an alkali species that acts as the mobile cationic charge carrier. For instance, glassy lithium oxochloride is of utmost current technological importance as the electrolyte of a proposed solid state battery [7]. More interesting from a fundamental point of view is the situation where the mobile alkali ions act as modifiers of a distinct glass backbone structure exemplified by alkali borate glasses: Here the end member borate oxide is a prototypical glass former in its own right, while the composition series $\left(A_{2} \mathrm{O}\right)_{x}\left(\mathrm{~B}_{2} \mathrm{O}_{3}\right)_{100-x}$, with $A$ an alkali metal, displays a pronounced vitrification tendency up to $x \approx 50$. A corresponding structure model is illustrated in Fig. 1. Various physical properties of binary alkali borate glasses show a strong dependence on composition and exhibit distinct extrema for each quantity, an effect called the borate anomaly [8].

Specifically, the diffusivity of the alkali species increases by orders of magnitude with growing $x$ due to the increased availability of diffusion paths. Surprisingly, in a mixed alkali borate glass $\left(A_{2} \mathrm{O}\right)_{x}\left(A_{2}^{\prime} \mathrm{O}\right)_{x^{\prime}}\left(\mathrm{B}_{2} \mathrm{O}_{3}\right)_{100-x-x^{\prime}}$, the diffusivity (amongst other materials properties) displays the so-called mixed alkali effect [12,13], where the diffusivities of $A$ and $A^{\prime}$ are given rather by $x$ and $x^{\prime}$, respectively, than by their sum. The most convincing explanation for this behavior seems to be the assumption of fixed and distinct sets of sites that 


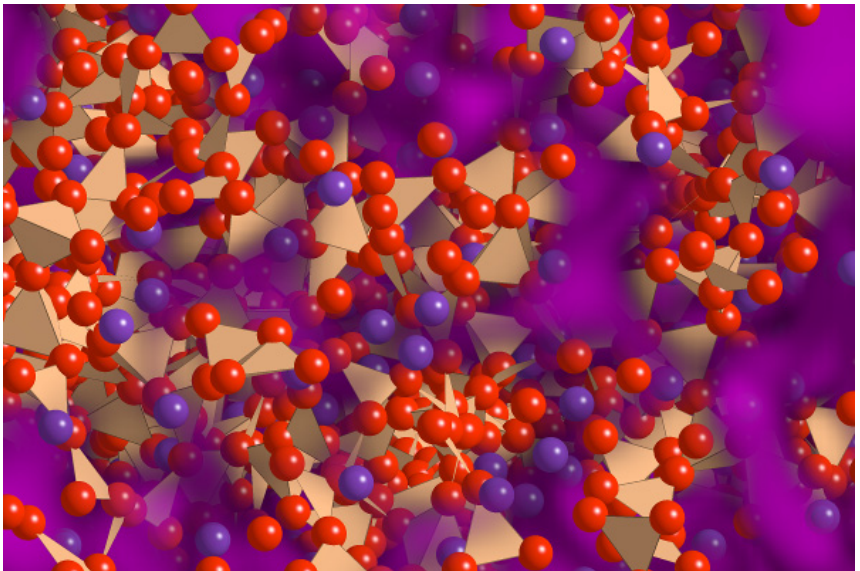

FIG. 1. Illustration of $\left(\mathrm{Rb}_{2} \mathrm{O}\right)_{30}\left(\mathrm{~B}_{2} \mathrm{O}_{3}\right)_{70}$ configuration emphasizing triangular or tetrahedral configuration of boron atoms (not shown) with respect to red oxygen atoms. The violet rubidium atoms are predominantly situated in the voids of the borate backbone, represented by the diffuse purple regions. The configuration was simulated with a potential adapted from Ref. [9].

are visited by the $A$ and $A^{\prime}$ atoms during their random walk through the structure. The concept of fixed sites in amorphous materials can already be found in early works by Haven and Verkerk [14] as well as many later works [15-17]. Although the models are somewhat different, all of these works include fixed alkali sites at least on certain timescales, an assumption that for reasons discussed above has not yet been directly studied experimentally.

The available knowledge on alkali dynamics rests mainly on tracer diffusion and conductivity measurements $[18,19]$. Assuming that the charge carriers perform independent random walks, the Nernst-Einstein relation strictly connects their contribution to the conductivity with their diffusivity. However, the increase of diffusivity with increasing alkali content together with the assumption of fixed sites implies correlation in the alkali movement. In the extreme case, the concept of vacancy diffusion familiar from crystalline systems results [20]. In this case, all possible alkali sites are occupied save for one vacant site, whose random motion on the alkali site network leads to diffusion of the alkali ions as accessible by tracer techniques. On the other hand, the conductivity results from the movement of the charged defect [21]. The random walks of the atoms and the vacancy in general have different correlation factors, which leads to a violation of the NernstEinstein relation, quantified by a Haven ratio different from one.

For a complete picture of diffusive dynamics in single alkali borate glasses, in our view the following three issues are critical: First, the degree of connectivity of the alkali site network-does it consist of essentially onedimensional chains [21] or does it correspond to a higherdimensional topology? Further, how many of the alkali sites are occupied-is the view of independent vacancies at low concentration correct, or are a significant fraction of possible sites unoccupied at any instant in time [22], or is even an interstitialcy model [16] the correct picture? And, finally, on which timescale does the alkali site network itself evolve- the

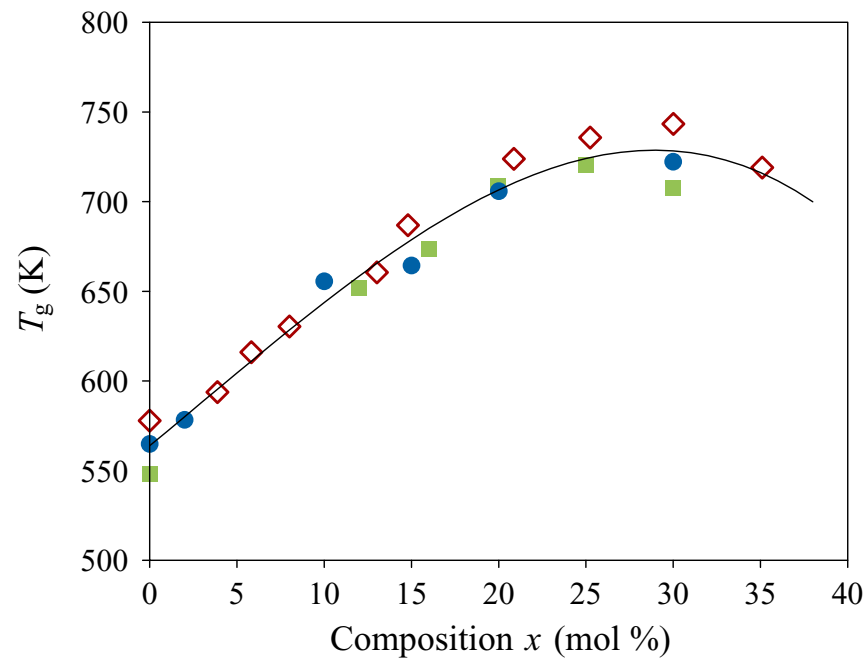

FIG. 2. Glass transition temperatures: Filled blue circles are values from present paper, empty red diamonds taken from Ref. [10], empty green squares from Ref. [11].

notion of sites that serve as stepping stones for the diffusing ions implies a stability on longer timescales than ionic diffusion, but how does it compare to the structural timescale of the borate backbone?

In this paper, we report on measurements of ionic conductivity as well as atomic-scale $\mathrm{x}$-ray correlation spectroscopy (aXPCS) on rubidium borate glasses. While the former extend the range of available data and serve to guarantee the comparability of our results to previous investigations, it is mainly the direct access to the density fluctuations on atomic scales in aXCPS that allows us to draw conclusions on the alkali site occupancy and stability that have hitherto not been experimentally accessible. Specifically, the most plausible interpretation of our experimental results is that ionic diffusion and conductivity proceeds via the movement of defects over a network of alkali sites, much like diffusion in crystalline matter. Defect densities are low, and in particular we do not find any indications for a distinct timescale pertaining to the evolution of the alkali site configuration. In this sense, diffusive dynamics in these systems are simpler than hitherto thought.

\section{EXPERIMENTAL METHODS}

\section{A. Sample preparation and characterization}

Rubidium borate glasses $\left(\mathrm{Rb}_{2} \mathrm{O}\right)_{x}\left(\mathrm{~B}_{2} \mathrm{O}_{3}\right)_{100-x}$ with compositions $x$ of $10,15,20$, and $30 \mathrm{~mol} \%$ have been prepared. The chemically pure materials were mixed and melted in alumina crucibles in an electrically heated muffle furnace at temperatures well above the respective glass transitions. The duration of melting was at least one hour depending on the sample composition. Successively, the samples were melt quenched to room temperature. All samples have been further annealed at temperatures slightly below the respective glass transitions over times of usually 72-96 hours. Being strongly hygroscopic, the samples were stored in a dry atmosphere at all times. The densities have been determined by their buoyancy in decahydronaphtalene to be $2.23,2.39,2.50$, and $2.84 \mathrm{~g} / \mathrm{cm}^{3}$ 

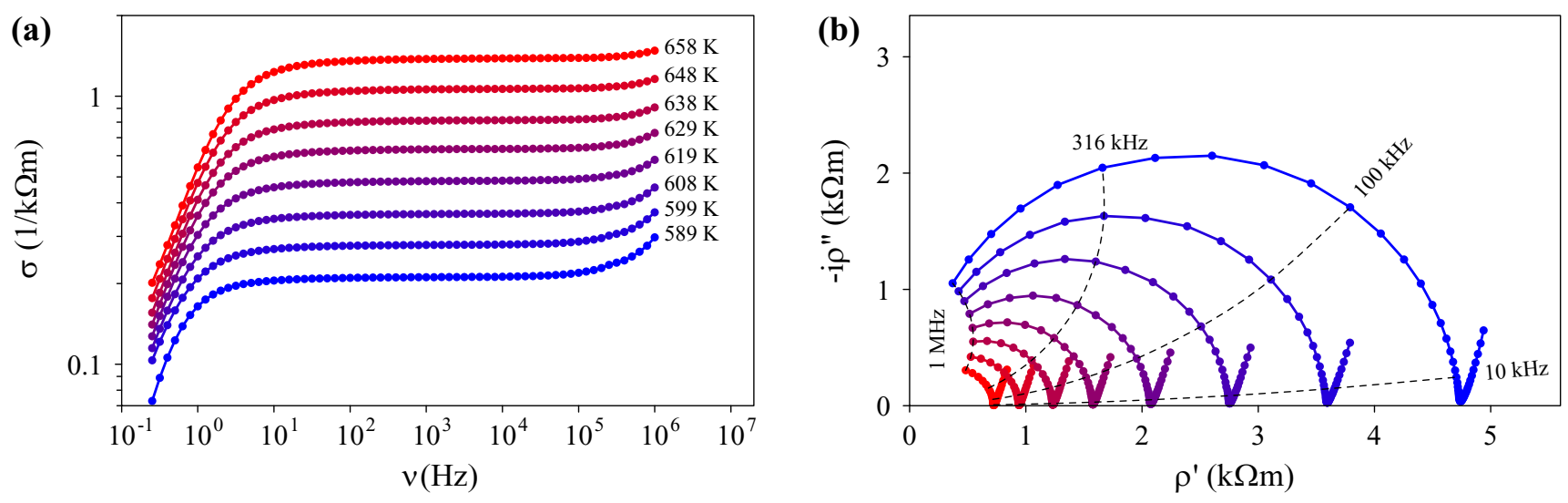

FIG. 3. Complex impedance spectra. (a) Real part of the conductivity versus the frequency for $\left(\mathrm{Rb}_{2} \mathrm{O}\right)_{30}\left(\mathrm{~B}_{2} \mathrm{O}_{3}\right)_{70}$. Different colors from blue to red represent different temperatures from $589 \mathrm{~K}$ to $658 \mathrm{~K}$. (b) Cole-Cole plot of the real part (horizontal axis) and imaginary part (vertical axis) of the frequency dependent specific resistivity for $\left(\mathrm{Rb}_{2} \mathrm{O}\right)_{30}\left(\mathrm{~B}_{2} \mathrm{O}_{3}\right)_{70}$, with the same color coding as in (a). Dashed lines are isofrequency lines.

for $x$ equal to $10,15,20$, and $30 \mathrm{~mol} \%$, respectively, in good agreement with Refs. [11,23].

Glass transition temperatures have been determined by differential scanning calorimetry using a Netzsch DSC 204 Phoenix calorimeter. To erase effects of thermal history, at least two heating-cooling cycles have been performed with a rate of $20 \mathrm{~K} / \mathrm{s}$. The inflection points in the second heating cycle are reported in Fig. 2, together with literature values on the variation of the glass transition temperature with composition $[10,11]$.

\section{B. Impedance spectroscopy}

Impedance spectroscopy gives information on the resistive and capacitive properties of materials by applying an alternating electric field. An ionic conductor can be seen as a parallel circuit of a resistor (describing charge flux due to diffusive ionic motion) and a capacitor (pertaining to the dielectric response of the charged structural components of the glass), giving the familiar semicircular Cole-Cole plots [see Fig. 3(b) for an example]. The intercept of the descending leg with the horizontal axis in principle gives the zero-frequency limit of the conductivity $\sigma_{\mathrm{dc}}$. Actually, at very low frequencies ionic accumulation and depletion at the electrodes correspond to an additional large capacitance in series to the original circuit that leads to a setup-dependent increase in the imaginary part of the impedance and thus to an apparent decrease in the real part of the conductivity [see Fig. 3(a)], which explains the need to perform measurements at alternating current. The plateau in the real part of the conductivity representative of $\sigma_{\mathrm{dc}}$ pertains to macroscopic diffusion of the charge carriers, while the discrete nature of ionic jumps visible at higher frequencies leads to a further increase in conductivity [24].

\section{Experimental details}

For the experiments measuring the charge diffusion coefficients $D_{\sigma}$, the samples were prepared as circular discs with thicknesses of $1 \mathrm{~mm}$ and cross-sectional diameters of $20 \mathrm{~mm}$. The two parallel planar cross sections were sputter-coated with 100 -nm gold layers serving as electrical contacts for the measurements. Fixing the samples between two platinum contact plates within a nitrogen-purged silica glass, the system was placed into a tube furnace for heating.

Complex impedance spectra $Z^{*}(\omega)$ were measured using an Alpha-A High Performance Modular Measurement System, built by Novocontrol Technologies, over a frequency range reaching from $0.1 \mathrm{~Hz}$ up to $1 \mathrm{MHz}$ with a frequencyindependent ac amplitude of $100 \mathrm{mV}$. Spectra were recorded from about $20 \mathrm{~K}$ below the glass transition temperature $T_{\mathrm{g}}$ toward lower temperatures.

\section{Results}

To illustrate the measurements, the impedance spectra for $\left(\mathrm{Rb}_{2} \mathrm{O}\right)_{30}\left(\mathrm{~B}_{2} \mathrm{O}_{3}\right)_{70}$ at a selection of temperatures are shown in Fig. 3. The full datasets are available for download [25]. Values for the low-frequency conductivity $\sigma_{\mathrm{dc}}(T)$ have been extracted by modeling the complex impedance with its ideal semicircular behavior and are plotted in Fig. 4. At temperatures sufficiently below the glass transition, the values show thermally activated behavior. Interestingly, the glass transition, which a priori could be expected to be of relevance only for structural aspects of the glass, also affects the conductivity, corresponding to a strong additional increase in conductivity in the region of some $50 \mathrm{~K}$ below the respective glass transitions, with a comparatively abrupt transition.

In the low-temperature regime, the data can be fitted with the Arrhenius expression

$$
\sigma_{\mathrm{dc}}(T) T=C_{0} \exp \left(-E_{\mathrm{A}} / k_{\mathrm{B}} T\right) .
$$

For the case of $\left(\mathrm{Rb}_{2} \mathrm{O}\right)_{30}\left(\mathrm{~B}_{2} \mathrm{O}_{3}\right)_{70}$, the composition where the low-temperature regime is best accessible in our data, the corresponding activation energy would be $E_{\mathrm{A}}=0.97 \mathrm{eV}$. This value is significantly different from the $0.875(3) \mathrm{eV}$ given in Ref. [11] for the same system (the corresponding data points are reproduced in Fig. 3). However, it has been recognized that the ionic conductivities in alkali borate glasses typically display a positive curvature in an Arrhenius plot [26-28]. Note that this behavior is distinct from the curved viscosity of fragile glasses captured by the Vogel-Fulcher-Tammann equa- 


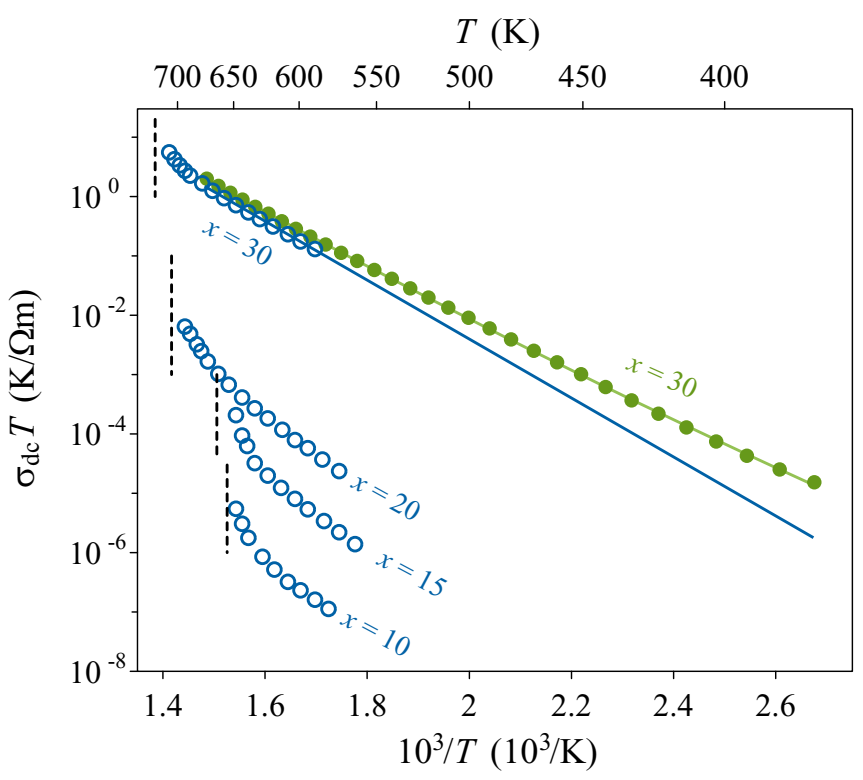

FIG. 4. Arrhenius plot of the product of the low-frequency conductivity $\sigma_{\mathrm{dc}}$ and the temperature $T$ for different rubidium borate glasses $\left(\mathrm{Rb}_{2} \mathrm{O}\right)_{x}\left(\mathrm{~B}_{2} \mathrm{O}_{3}\right)_{100-x}$. Empty blue circles are values from the present paper with fit according to Eq. (1), filled green symbols are from Ref. [11] for $\left(\mathrm{Rb}_{2} \mathrm{O}\right)_{30}\left(\mathrm{~B}_{2} \mathrm{O}_{3}\right)_{70}$ and the green upward curved line shows the respective fit with Eq. (2). The dashed vertical lines mark the respective glass transition temperatures.

tion with positive $T_{0}$, which would correspond to a negative curvature [4].

The observed positive curvature has been proposed to be a consequence of a distribution of activation energies for the distinct diffusing atoms due to the inequivalent local configurations. Assuming Gaussian distributions of activation energies with a standard deviation $\sigma$ around $E_{\mathrm{A}}^{0}$ leads to an expression of

$$
\sigma_{\mathrm{dc}}(T) T=C_{0}^{\prime} \exp \left(-E_{\mathrm{A}}^{0} / k_{\mathrm{B}} T\right) \exp \left(\sigma^{2} / 2\left(k_{\mathrm{B}} T\right)^{2}\right)
$$

in the limit $\sigma \ll E_{\mathrm{A}}^{0}[29]$. Fitting this expression to the data from Ref. [11] yields $E_{\mathrm{A}}^{0}=1.15 \mathrm{eV}$ and $\sigma=0.11 \mathrm{eV}$, which gives a slope at $630 \mathrm{~K}$ that would correspond to an activation energy of $E_{\mathrm{A}}=0.93 \mathrm{eV}$ via Eq. (1). Thus, the slopes of the data sets are consistent, and we assign the small deviation in magnitude to slightly different actual compositions or thermal histories.

\section{Atomic-scale x-ray photon correlation spectroscopy}

The method of choice to access slow structural dynamics at the atomic scale in disordered media is atomic-scale $\mathrm{x}$-ray photon correlation spectroscopy (aXPCS). The idea of studying the temporal evolution of density fluctuations in reciprocal space, which are directly accessible as speckles in the scattering pattern when utilizing coherent incident radiation, was first proposed with the availability of lasers as coherent optical light sources [30] and is today a standard experimental tool known as dynamic light scattering, intensity fluctuation spectroscopy, or photon correlation spectroscopy [31]. With the initial demonstration of coherent $\mathrm{x}$-ray scattering [32] and the construction of dedicated synchrotron beamlines, mainly soft matter dynamics in the nanometer range has been studied [33-35]. Finally, since the requirements on coherence and scattering efficiency for studying atomic-scale dynamics had been met [36,37], the method has been applied for studying the diffusive behavior of atoms in crystalline [38,39] and amorphous media [40-45].

\section{Principles}

In principle, in an $\mathrm{x}$-ray scattering experiment, the recorded intensity is given strictly by the absolute square of the Fourier transform of the sample's electron density. In particular, $a$ priori no averaging is involved, so the sample's microstate is directly probed. For a disordered sample, this implies a graininess of the scattering pattern in the diffuse regime characteristic of the specific arrangement of the atoms and thus electrons, called a speckle pattern. The reason for the absence of such graininess in conventional $x$-ray scattering experiments lies in the finite monochromaticity and imperfect collimation of the incident radiation as well as a finite positional resolution of the detectors. Thus, the recorded photons at a given nominal position on the detector actually correspond to slightly different wave-vector transfers $\vec{q}$, and their incoherent addition leads to a washing-out of the interference pattern, corresponding to conventional $\mathrm{x}$-ray scattering being sensitive only to averages over microstates [46]. The experimental parameters corresponding to the detected intensity being averaged over given volumes in reciprocal space can be translated to real-space longitudinal and transversal coherence lengths (again related by a Fourier transformation to the reciprocal space averaging volume), and the condition for a good speckle visibility is that the illuminated sample volume not be much larger than the corresponding coherence volume [47]. Note that this condition is equivalent to the Bragg peaks in single-crystal scattering displaying single-slit scattering fringes.

As atomic rearrangements take the sample from one microstate to the next, the corresponding scattering pattern changes. To be specific, as it is the spatially varying electron density that determines the scattering pattern, a hypothetical exchange of two atoms of the same element does not have an effect. In this sense, aXPCS is equivalent to quasielastic neutron scattering with coherent scatterers, which sees the temporal fluctuations in intensity as a broadening in energy of the elastic line and thus is sensitive specifically to fast dynamics. In the following, we will term the fundamental dynamic events that do lead to a temporal evolution of the scattering pattern as structural rearrangements.

Different from the optical regime, where lasers are abundant coherent light sources, in the x-ray regime photon densities are much smaller. While the newly available $\mathrm{x}$-ray free electron lasers are inherently semicoherent sources, at third-generation synchrotron facilities as used here, coherent conditions can be achieved only by monochromatization and collimation, thus cutting out a comparatively small volume of photon phase space. In any case, in the diffuse regime at wave-vector transfers corresponding to atomic scales, the scattered intensity is very small. In contrast to the small-angle scattering case, where observable speckle patterns can indeed be recorded [47], here only single photons are detected. By 
averaging over the pixels of a multidetector and absolute time, the resulting Poisson noise can be reduced to obtain statistically significant autocorrelation functions: Specifically, with $n_{i, t}$ being the counted photons in pixel $i$ and frame $t$ for a total of $N$ pixels and $T$ frames, the experimental normalized autocorrelation function is computed as

$$
g^{(2)}(\vec{q}, \Delta t)=\frac{N \sum_{i} \sum_{t=1}^{T-\Delta t} n_{i, t} n_{i, t+\Delta t}}{\sum_{t=1}^{T-\Delta t} \sum_{i} n_{i, t} \sum_{j} n_{j, t+\Delta t}},
$$

where the angle of the detector with respect to the incident beam together with the wavelength determine the corresponding wave-vector transfer $\vec{q}$.

Under mild assumptions that correspond to the illuminated volume being much larger than the scale of spatial fluctuations [48], the experimental auto-correlation function is essentially equivalent to the (coherent) intermediate scattering function $I(\vec{q}, \Delta t)$ familiar from the theory of inelastic neutron scattering [49],

$$
\left\langle g^{(2)}(\vec{q}, \Delta t)\right\rangle=1+A\left(\frac{I(\vec{q}, \Delta t)}{I(\vec{q}, 0)}\right)^{2},
$$

which in turn is the spatial Fourier transform of van Hove's pair correlation function $G(\Delta \vec{r}, \Delta t)$ [50]. The coherence factor, optical contrast or speckle visibility $[47,51,52] A$ varies between 0 and 1 and quantifies how well the coherence requirements are met. It is a function of the experimental conditions such as monochromaticity, wave-vector transfer, and sample dimensions, but it is independent of the sample dynamics.

The intermediate scattering function $I(\vec{q}, \Delta t)$ quantifies the agreement between speckle patterns taken a given time delay $\Delta t$ apart, and thus equivalently the agreement of the corresponding Fourier components of the sample's electron density. By definition, the normalized intermediate scattering function $I(\vec{q}, \Delta t) / I(\vec{q}, 0)$ is unity for $\Delta t=0$. At short timescales in the picosecond regime, it would display an oscillatory behavior due to phonon dynamics, followed by a monotonous decay on the timescale of diffusive dynamics. With the temporal resolution of aXPCS, only the diffusive part is visible. In the simplest case, such as if the scatterers perform independent memoryless random walks, the diffusive decay is a simple exponential with a characteristic decay rate constant $\Gamma(\vec{q})$ [53]. Due to the inequivalent local configurations in a glass, in a given configuration some structural features will be longer lived than others, which can be expected to correspond to a distribution of decay rates. Phenomenologically, such behavior can be captured by the Kohlrausch-Williams-Watts expression

$$
I(\vec{q}, \Delta t)=I(\vec{q}, 0) \exp \left(-\{\Gamma(\vec{q}) \Delta t\}^{\beta}\right),
$$

with shape parameters $\beta<1$, corresponding to so-called stretched exponential decays [54].

\section{Experimental details}

The aXPCS samples have been prepared by cutting slabs from the annealed ingots with a low-speed diamond saw. These slabs were then ground to a thickness of $200 \mu \mathrm{m}$, and finally holes in the shapes of spherical segments were

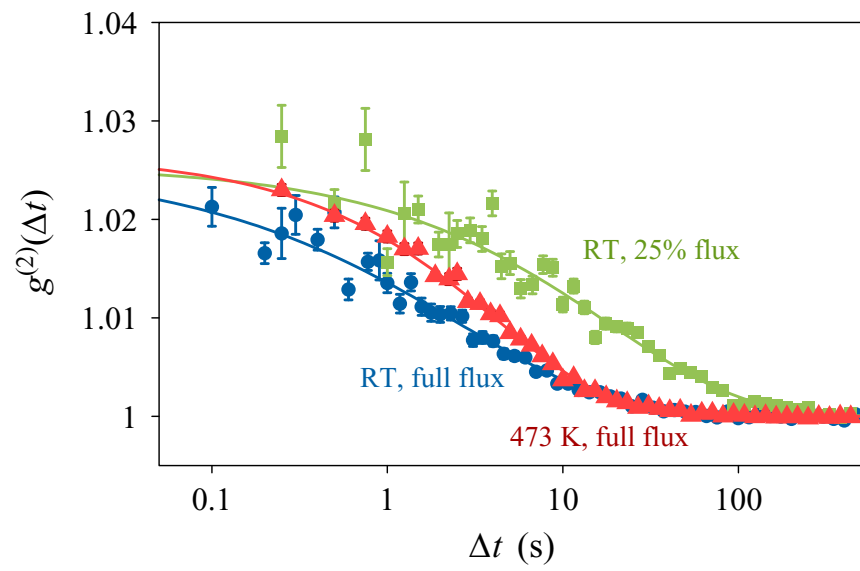

FIG. 5. Intensity autocorrelation functions for $\left(\mathrm{Rb}_{2} \mathrm{O}\right)_{30}\left(\mathrm{~B}_{2} \mathrm{O}_{3}\right)_{70}$ glass at room temperature for full flux (blue circles) and with flux attenuated to $25 \%$ (green squares), as well as at $473 \mathrm{~K}$ and full flux (red triangles).

excavated by a dimpling grinder, aiming for a thickness at the thinnest spot of $50 \mu \mathrm{m}$. This was done to be able to choose the effective sample thickness at the experiment by scanning the beam over the excavation. The thicknesses at the actual measured spots were calculated from the measured beam attenuation in transmission, using the known absorption lengths. During the measurements, the actual position on the sample was shifted by a few micrometers at regular intervals to preclude possible beam damage. Thus, the thicknesses varied in the range $45-51 \mu \mathrm{m}$.

The aXPCS measurements were conducted at beamline P10 of PETRA III, with auxiliary measurements performed at ID10 at the ESRF. At P10, a coherent setup with a Si(111) channel-cut monochromator at $13 \mathrm{keV}$ and a compound refractive lens system giving a focus size (FWHM) of about $3 \times 2 \mu \mathrm{m}^{2}(\mathrm{~h} \times \mathrm{v})$ was used, corresponding to dose rates of about $0.7 \mathrm{eV}$ absorbed per atom and second. The scattered radiation was detected with an EIGER $4 \mathrm{M}$ pixel detector (75 $\mu \mathrm{m}^{2}$ pixel size) at a distance of $1.80 \mathrm{~m}$, using an energy threshold of $10.01 \mathrm{eV}$. Frame exposure times were in the range of $0.1-1.5 \mathrm{~s}$, depending on the amount of scattered photons. Samples were mounted in a resistively heated custom vacuum sample furnace. Temperature stability was better than about $0.2 \mathrm{~K}$ at elevated temperatures, and the vacuum was below $10^{-5}$ mbar for all measurements. The intensity autocorrelation function was determined at various temperatures but at a fixed wave-vector transfer $q$, chosen to match the first glass peak in the static scattering function of vitreous $\left(\mathrm{Rb}_{2} \mathrm{O}\right)_{30}\left(\mathrm{~B}_{2} \mathrm{O}_{3}\right)_{70}$ at $q_{\max }=1.89 \AA^{-1}$. Typical autocorrelation functions are illustrated in Fig. 5. The full data sets are available for download [55]. The decay rates were obtained by fitting Eq. (5) with a free stretching parameter $\beta$ of about 0.5 at low temperatures, increasing toward unity around $600 \mathrm{~K}$. A table of the resulting parameters is given in the Supplemental Material [56].

$\mathrm{X}$-ray scattering experiments in a laboratory $\mathrm{x}$-ray setup have been performed before and after the aXPCS experiments to ensure that no crystallization had occurred. Further, the 
two-time auto-correlation function,

$$
C\left(t_{1}, t_{2}\right)=\frac{N \sum_{i} n_{i, t_{1}} n_{i, t_{2}}}{\left(\sum_{i} n_{i, t_{1}}\right)\left(\sum_{i} n_{i, t_{2}}\right)},
$$

was computed for every measurement to check against dynamical heterogeneities-for steady-state dynamics, $C\left(t_{1}, t_{2}\right)$ depends only on the time delay $\Delta t=t_{2}-t_{1}$ and reduces to the conventional autocorrelation function Eq. (3).

\section{DISCUSSION}

\section{A. Timescales of diffusive dynamics}

In the low-frequency limit, the connection between conductivity and diffusion of independently moving charge carriers is given by the Nernst-Einstein relation. Let us assume a constant applied electrical field and consider the corresponding steady-state solution of carrier concentration $n(x)$ : The electrical current density that would result from the electrical field alone $\vec{j}_{\mathrm{el}}=\sigma \vec{E}=-\sigma V^{\prime}(x)$ and the current density due to charge carrier diffusion $\vec{j}_{\text {diff }}=-q D_{\sigma} n^{\prime}(x)$ have to cancel, where $\vec{E}$ is the electric field, $V(x)$ the electric potential, $q$ the charge per carrier, and $n(x)$ the number density of the carriers. With the number density given by the Boltzmann factor $n(x) \propto \exp \left(-q V(x) /\left(k_{\mathrm{B}} T\right)\right)$, the relation

$$
\sigma=\frac{q^{2} n}{k_{\mathrm{B}} T} D_{\sigma}
$$

follows. The number density of the $\mathrm{Rb}^{+}$ions results from the above-quoted mass density as $n=9.8 \times 10^{27} \mathrm{~m}^{-3}$ for $\left(\mathrm{Rb}_{2} \mathrm{O}\right)_{30}\left(\mathrm{~B}_{2} \mathrm{O}_{3}\right)_{70}$. Note that, as discussed in the Introduction, the charge diffusivity is typically faster than the corresponding tracer diffusivity - the Haven ratio in this system has been found to be about 0.24 at room temperature and ambient pressure [58].

In contrast to the discrete atomic jumps in crystalline matter that lead to a characteristic behavior of the $q$-dependent decay rate constants $\Gamma(q)$ [38], we have shown in a previous publication that there is no evidence of such effects of the jump geometry on $\Gamma(q)$ in alkali borates [59]. Rather, its behavior is reproduced by assuming the diffusing entities to perform Brownian motion composed of infinitesimal jumps, which by itself would correspond to the decay rates being proportional to $q^{2}$, modified by the atom-atom interactions that determine the realized network structure and thus the structure factor $S(q)$. We termed the resulting model interacting Brownian motion, and we have found its prediction

$$
\Gamma(q)=q^{2} D_{\Gamma} / S(q)
$$

to be able to describe the data satisfactorily. In the present case, the height of the structural peak is about 2, giving $D_{\Gamma}=$ $5.6 \times 10^{-21} \mathrm{~m}^{2} \cdot \Gamma\left(q_{\max }\right)$.

The third independent handle on atomic-scale diffusive dynamics is afforded by the behavior of the macroscopic viscosity. The Stokes-Einstein equation

$$
D_{\eta}=\frac{k_{\mathrm{B}} T}{6 \pi \eta r}
$$

relates the translational diffusion constant of a spherical particle of radius $r$ in a viscous medium to the viscosity $\eta$. As it

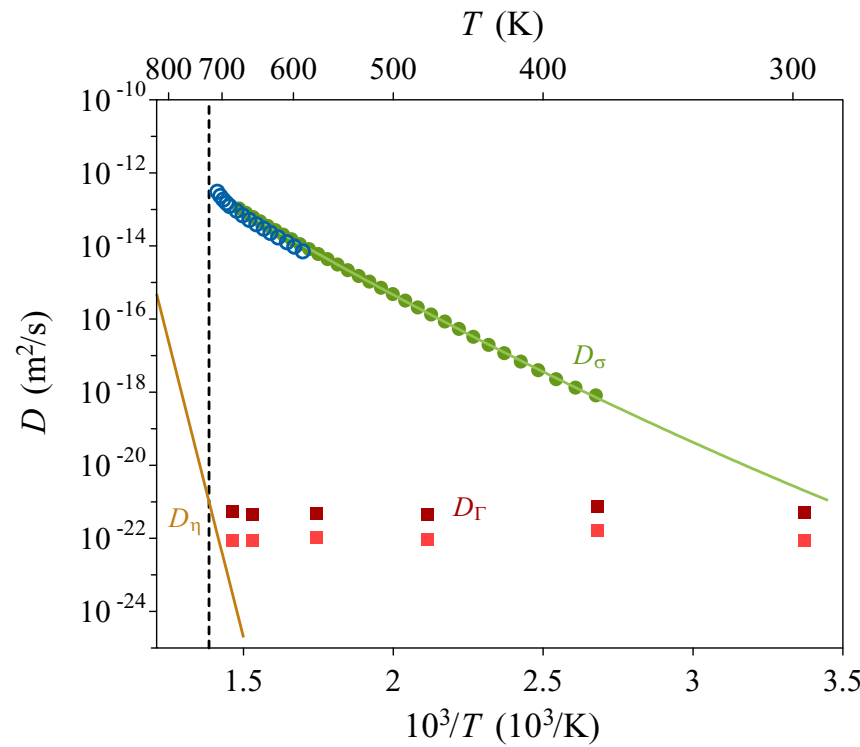

FIG. 6. Diffusion data for $\left(\mathrm{Rb}_{2} \mathrm{O}\right)_{30}\left(\mathrm{~B}_{2} \mathrm{O}_{3}\right)_{70}$ : Charge diffusion coefficients $D_{\sigma}$ determined in this paper (empty blue points) and from Ref. [11] (filled green points) with extrapolation according to Eq. (2) (green line), aXPCS diffusion coefficients $D_{\Gamma}$ obtained for full x-ray flux (dark red squares) and with flux attenuated to $25 \%$ (light red squares), as well as viscosity diffusion coefficient $D_{\eta}$ fitted to values measured around $700 \mathrm{~K}$ in Ref. [57] (brown line). The dashed vertical line marks the glass transition temperature.

is clearly the borate backbone, i.e., the $\mathrm{B}-\mathrm{O}$ bonds, that are responsible for said viscosity, the diffusion constant $D_{\eta}$ will be representative for the boron-oxygen dynamics. Arguably, the smallest meaningful structural units in terms of diffusive dynamics are the $\mathrm{BO}_{3}$ triangles or $\mathrm{BO}_{4}$ tetrahedra. With a characteristic B-O distance of $1.48 \AA$ [60], we assume a viscous radius of $r=2.5 \AA$ for these structural units. While it is clear that the macroscopic dynamical behavior's extrapolation cannot be expected to describe dynamics at the atomic scale perfectly, we nevertheless see no reason to expect deviations larger than a factor of 2 .

Figure 6 shows the temperature-dependent values of $D_{\sigma}$ and $D_{\Gamma}$ as obtained here and in Ref. [11] for $\left(\mathrm{Rb}_{2} \mathrm{O}\right)_{30}$ $\left(\mathrm{B}_{2} \mathrm{O}_{3}\right)_{70}$ together with values of $D_{\eta}$ determined from the viscosities given in Ref. [57] for the slightly different composition $\left(\mathrm{Rb}_{2} \mathrm{O}\right)\left(\mathrm{B}_{2} \mathrm{O}_{3}\right)_{2}$. The fact that there is a large discrepancy between $D_{\sigma}$ and $D_{\eta}$ (corresponding to the alkali ions and the borate backbone, respectively) of about nine orders of magnitude at the glass transition is not surprising, as this is the essence of alkali borate glasses being superionic conductors. The intention of the decoupling index is to quantify just the ratio of these two quantities: For simplicity, an approximate expression using a fixed viscosity relaxation rate is typically used [61], and values of about ten orders of magnitude or more are found in comparable systems [62,63].

The apparently temperature-independent $D_{\Gamma}$ necessitates more comment: Recently, it has been recognized that, generally in insulating glasses [45,64] and specifically in alkali borates [59], low-temperature dynamics as accessible in aXPCS experiments are driven by transient radiolysis of the covalent bonds and thus are proportional to the absorbed dose 
rate. Indeed, the autocorrelation functions in Fig. 5 and the fitted decay rates in Fig. 6 show that reducing the beam intensity by a factor of 4 compared to the full beam lead to a corresponding slowing of dynamics. Thus, our probed $D_{\Gamma}$ has to be interpreted as an upper bound on the intrinsic, temperaturedriven dynamics as would be accessible in a hypothetical aXPCS experiment in the absence of an accelerating effect of absorbed radiation on the sample dynamics.

Quantitatively comparing the distinct notions of diffusivity as a function of temperature in Fig. 6 suggests strongly that dynamics as measured by aXPCS neither closely follow ionic charge diffusion (and thus tracer diffusion) dynamics nor are intermediate between ionic dynamics and structural dynamics of the borate backbone as quantified by $D_{\eta}$, but most likely are equal to the latter. Unfortunately, large-scale relaxations begin to dominate the aXCPS signal at higher temperatures, while reducing the incident flux further is not feasible with the concomitant reduction in statistical precision due to photon noise. Thus, it was not possible to directly see the expected merging of $D_{\eta}$ and $D_{\Gamma}$ at temperatures at or slightly above the glass transition at $720 \mathrm{~K}$ [45]. Still, we are confident of the equivalence of the intrinsic $D_{\Gamma}$ with $D_{\eta}$ : The spatial configurations' dynamics sampled by aXPCS cannot conceivably be significantly slower than the rearrangements corresponding to viscous flow, while there is hardly room left on the faster side.

Having connected the dynamics accessed by aXCPS to the timescales displayed by the borate backbone, it remains to discuss the question of which kind of dynamics aXPCS is actually sensitive to (that is, to which component of this ternary system the structural rearrangements as defined previously pertain to). After all, if the scattering pattern were given mainly by the configuration of the borate backbone, the point concluded above would hardly be surprising.

\section{B. Weights of distinct contributions to the aXPCS signal}

Consider the scattered amplitude to be composed of two partial amplitudes $A_{X}(t)$ and $A_{Y}(t)$ modeled as independent complex-valued Gaussian processes with different second moment and temporal behavior. Writing the unnormalized intensity autocorrelation function

$$
G^{(2)}(\Delta t)=\langle I(t) I(t+\Delta t)\rangle
$$

with the scattered intensity $I(t)$ being the absolute square of the total amplitude $A(t)=A_{X}(t)+A_{Y}(t)$, an elementary calculation shows that

$$
G^{(2)}(\Delta t)=\left(\left\langle I_{X}\right\rangle+\left\langle I_{Y}\right\rangle\right)^{2}+\left(G_{X}^{(1)}(\Delta t)+G_{Y}^{(1)}(\Delta t)\right)^{2},
$$

where $G_{X}^{(1)}(\Delta t)$ is the unnormalized first-order correlation function or amplitude correlation function

$$
G_{X}^{(1)}(\Delta t)=\left\langle A_{X}(t) A_{X}^{*}(t+\Delta t)\right\rangle
$$

for species $X$ (and analogously for $Y$ ), with $G_{X}^{(1)}(0)=\left\langle I_{X}\right\rangle$. Conceptually, this is a consequence of Isserlis' theorem [65], with the absence of cross terms being due to the assumed independence of the two processes. As discussed above, partial coherence will decrease the contribution of the nontrivial second term, while the relative weights of the two processes are unaffected.
For the case at hand, we divide the total intensity into contributions due to the $\mathrm{Rb}^{+}$ions on the one hand and due to the oxygen and boron atoms on the other hand. In the simplest approach, we assume a total absence of order, so the contributions are given by the products of the respective number densities and scattering cross sections, which we set equal to the squared atomic number $Z^{2}$. This can be easily seen to give relative intensities of $I_{\mathrm{Rb}}=0.813$ and $I_{\mathrm{B}, \mathrm{O}}=0.187$. Note that this strong contribution found for the $\mathrm{Rb}^{+}$ions is even a very conservative estimate, as computing the partial structure factors from simulated configurations [9] with the actual atomic form factors implies only $8.4 \%$ of the total scattering at the position of measurement $q_{\max }$ to result from the B-B, O-O, and $\mathrm{B}-\mathrm{O}$ terms. The reason for the strong additional reduction of the non- $\mathrm{Rb}^{+}$terms is that the structural peak in this system is due to the characteristic $\mathrm{Rb}$ - $\mathrm{Rb}$ distances, while the maxima in the other partial structure factors are at different positions.

\section{Microscopic picture}

The main consequence to be drawn from our experimental data is the stability of the $\mathrm{Rb}^{+}$ions' configuration up to the timescales of the borate backbone's structural rearrangements. This follows from the following chain of argumentation: First, we do not have any indications of dynamics on timescales shorter than the experimentally accessible time window, which would result in a decrease of apparent contrast [66], beyond phononlike oscillations as conventionally modeled by the Debye-Waller factor. Thus, on sub-seconds timescales (cf. Fig. 5) the configuration of scatterers is essentially static. According to the previous section, the scattered radiation is dominated by the $\mathrm{Rb}^{+}$ions, so in particular their configuration is unchanged. At high temperatures, the decay of correlations on the timescale of seconds does coincide with the extrapolation from macroscopic viscosity, while at lower temperatures it is masked by the beam perturbation.

The simplest scenario that can explain the decoupled dynamics of the ions and the borate backbone, which arguably is the basic nontrivial experimental fact on diffusion in these systems, would be a picture where the ions perform an independent gaslike diffusion through the open glass network. Our results rather imply the opposite extremal picture to be appropriate, where the sites occupied by $\mathrm{Rb}^{+}$ions are stable in time, while the individual $\mathrm{Rb}^{+}$jump from site to site. This stability is to be understood not only in a qualitative sense as compact voids within the borate backbone in the sense of Ref. [22] but also as a constraint on the actual positions within these voids to displacements $\Delta r \lesssim 1 / q \approx 0.5 \AA$, where $q$ is the probed wave-vector transfer. Such a behavior has been seen in molecular dynamics simulations, e.g. in the analogous sodium silicate and lithium phosphate systems [67,68], but to our knowledge has never been concluded in a direct experimental investigation.

We want to explicitly discuss two direct consequences of this finding. First, it contradicts the site-memory assumption of the so-called dynamic structure model [17]: this model considers fixed alkali sites with individual adaptations for being occupied by a given alkali species, where the adaptations relax toward reinforcing the instantaneous occupation, with the memory relaxation rate being much faster than the 
persistence time of the glass structure. According to our results, beyond the ionic hopping rates there is only a single timescale, namely, the timescale of rearrangements of the glass network - the fitness of a given alkali site (or, rather, a given position in space being an alkali site at all) is directly linked to the glass network's configuration. Put in other words, the configuration of potential alkali sites will change only simultaneously with rearrangements in the borate network. According to the perhaps simplistic view of the glass structure being frozen below the glass transition, the corresponding timescale would go to infinity.

Further, it implies that ionic diffusion is driven by defects with low concentrations. It is commonly assumed that in the glass structure that freezes at the glass transition a few vacant alkali sites remain that are essentially energetically equivalent to the occupied sites. Molecular dynamics simulations would predict such frozen-in vacancy concentrations of a few percent in analogous systems $[22,68,69]$, but as extreme case it has also been proposed that the frozen-in defect concentration is essentially zero, necessitating thermal Frenkel defect generation [70]. The absence of intensity fluctuations in the scattered radiation on the ionic diffusion timescale allows us to put the experimental limit on unoccupied alkali sites conservatively at around $10 \%$, also considering the fact that a vacant site would lead to strong positional relaxations of the neighboring $\mathrm{Rb}^{+}$ions due to the (missing) Coulomb interactions, which would enhance the signal. Note that while this absence of fast fluctuations would also be consistent with spontaneous ring exchanges of ions unaided by any defect, such dynamical events would only contribute to tracer diffusivity but not to charge diffusivity and can thus be excluded on the grounds of a Haven ratio clearly below one [58].

Given the added insights obtained by our experiments as discussed above, we note that the actual nature of the fundamental events of ionic diffusion has to be regarded still as an open question. Specifically, the low Haven ratio of about 0.24 at ambient pressure, decreasing to 0.02 at a pressure of $6 \mathrm{kbar}$ which corresponds to a reduction in molar volume of only $2 \%$ [23], has been explained as an effect of synergy in migration energy if successive vacancy movements are close to collinear [58]. Clearly, this implies a collective nature of diffusive dynamics, where the moving vacancy defect is not localized to a single alkali site but spread over a number of sites, so the step of the vacancy defect by one site corresponds to $N$ ions performing each a movement on the order of $1 / N$ in close to collinear fashion. On the other hand, the widely accepted explanation of the increase in ac conductivity at high frequencies is in terms of well-defined discrete ionic jumps. Indeed, following Ref. [71] we can equate $v_{0}$, the frequency where the real part of the conductivity as given in Fig. 3(a) has doubled compared to the dc value, with the ionic jumping frequency. Taking $v_{0}=2.5 \mathrm{MHz}$ at $589 \mathrm{~K}$ and reading the corresponding charge diffusivity from Fig. 6 as $D_{\sigma}=7 \times 10^{-15} \mathrm{~m}^{2} / \mathrm{s}$ implies jump distances of about $1.3 \AA$, a value that is clearly in the correct order of magnitude.

Further, while our data show the stability of the $\mathrm{Rb}^{+}$site configuration, we do not have direct evidence for the details of the ions' dynamics on these sites or their actual microscopic arrangements. In particular, we cannot judge whether the sites are dynamically equivalent or not, and, connected to this issue, on the alkali sites making up conductive channels such as in Greaves' modified random network model [72]. Of course, the inequivalent local environments in the aperiodic glass arrangement prohibit an ideal dynamical equivalence of the sites, and in molecular dynamics simulations on lithium phosphate glass there indeed has been found a continuous distribution of site-resolved jump rates over at least three orders of magnitude at low temperatures [68]. In contrast, simulations on lithium borate glass have been interpreted in terms of two qualitatively different types of sites, where those adjacent to a nonbridging oxygen atom showed dynamics faster by about one order of magnitude [73]. Our results would now be also consistent with a model where the predominant majority of alkali sites belongs to the slow kind that do not contribute to diffusion, are always fully occupied and thus responsible for the temporal stability of the scattered intensity as observed here, while a small minority of sites, effectively invisible to aXPCS, would host ions as mobile as the $\mathrm{Ag}^{+}$ions in $\alpha$-AgI. In this sense, the concept of vacancylike defects as we have used it here would be inappropriate (we thank an anonymous referee for pointing this out). However, the decreased density of diffusing ions in this scenario together with a fixed ionic jump frequency $v_{0}$ as discussed above would necessitate jump distances that surpass typical atomic distances, thus rendering this extreme scenario quite implausible in our view.

Note that our previous finding that the $q$-dependent relaxation rates in a number of vitreous alkali borate systems including the present $\left(\mathrm{Rb}_{2} \mathrm{O}\right)_{30}\left(\mathrm{~B}_{2} \mathrm{O}_{3}\right)_{70}$ are best described by infinitesimal displacements [59] is not at variance with a model of ionic jumps on the order of some $\AA$ : As discussed earlier, fluctuations in the scattered radiation are due to structural rearrangements of the borate backbone and the interspersed alkali sites. This happens on much slower timescales than ionic diffusion, and the observed infinitesimal displacements can indeed be explained as the long-ranged elastic relaxations of the glass structure as response to a local topological rearrangement of the network [59].

Finally, we want to reiterate that our observed intensity fluctuation relaxation rates $\Gamma$ being due to beam-driven as opposed to thermal dynamics does not invalidate our conclusions. Our argument rests on the gap between the fast ionic dynamics as accessed by conductivity and the slow rate of structural rearrangements as probed by aXPCS. As the absorption of hard X-ray photons can conceivably only accelerate the dynamics and never slow it down, a hypothetical measurement of $D_{\Gamma}$ in the absence of beam perturbation would give much slower dynamics, so the values reported in Fig. 6 give a very conservative picture of the actual situation.

\section{SUMMARY}

In the Introduction, we stated three open questions with respect to alkali ion diffusivity and conductivity in glasses, viz., the topology of the arrangement of alkali sites, the actual occupation of these potential sites by ions, and the temporal stability of the sites. By measurements of electrical conductivity on a range of rubidium borate glasses and specifically aXPCS experiments on $\left(\mathrm{Rb}_{2} \mathrm{O}\right)_{30}\left(\mathrm{~B}_{2} \mathrm{O}_{3}\right)_{70}$ we have been able to answer the latter two of these issues: The absence of intensity fluctuations in the coherent scattering allows us to 
conclude that the configuration of $\mathrm{Rb}^{+}$ions is remarkably stable in time, implying that the concentrations of vacancy or interstitial defects are very low, and the arrangement of possible alkali sites themselves does evolve only on the timescales that describe also the borate backbone's dynamics. Thus, our report adds a significant new feature to the decoupling concept: it is not just that the alkali ions diffuse much faster through the system than the glass network evolves, but the discrepancy is instead between the diffusion of the individual alkali ions and the lifetime of the alkali sites.

\section{ACKNOWLEDGMENTS}

We thank M. Sprung, F. Westermeier, and the whole team of beamline P10 at PETRA III in Hamburg, and B. Ruta and F. Zontone at beamline ID10 at ESRF in Grenoble for their assistance. We acknowledge the support of A. Opitz of the TU Wien with conductivity measurements and H. Peterlik and P. Seebacher of the University of Vienna for support with auxiliary X-ray scattering experiments. This research was funded by the Austrian Science Fund (FWF): P28232-N36.
[1] G. Vogl and B. Sepiol, in Diffusion in Condensed Matter: Methods, Materials, Models, 2nd ed. edited by P. Heitjans and J. Kärger (Springer, Berlin, 2005), pp. 65-91.

[2] R. Terai and R. Hayami, J. Non-Cryst. Solids 18, 217 (1975).

[3] J. W. Haus and K. W. Kehr, Phys. Rep. 150, 263 (1987).

[4] C. A. Angell, K. L. Ngai, G. B. McKenna, P. F. McMillan, and S. W. Martin, J. Appl. Phys. 88, 3113 (2000).

[5] W. Dieterich and P. Maass, Chem. Phys. 284, 439 (2002).

[6] S. Hull, Rep. Prog. Phys. 67, 1233 (2004).

[7] M. H. Braga, C. M Subramaniyam, A. J. Murchison, and J. B. Goodenough, J. Am. Chem. Soc. 140, 6343 (2018).

[8] J. E. Shelby, Introduction to Glass Science and Technology, Royal Society of Chemistry Paperbacks (Royal Society of Chemistry, Cambridge, 1997).

[9] A. H. Verhoef and H. W. den Hartog, J. Non-Cryst. Solids 182, 235 (1995).

[10] W. Soppe, W. Ebens, and H. W. Den Hartog, J. Non-Cryst. Solids 105, 251 (1988).

[11] F. Berkemeier, S. Voss, Á. W. Imre, and H. Mehrer, J. NonCryst. Solids 351, 3816 (2005).

[12] D. E. Day, J. Non-Cryst. Solids 21, 343 (1976).

[13] Á. W. Imre, S. V. Divinski, S. Voss, F. Berkemeier, and H. Mehrer, J. Non-Cryst. Solids 352, 783 (2006).

[14] Y. Haven and B. Verkerk, Phys. Chem. Glasses 6, 38 (1965).

[15] M. Lax and H. Scher, Phys. Rev. Lett. 39, 781 (1977).

[16] S. R. Elliott, Solid State Ionics 27, 131 (1988).

[17] A. Bunde, M. D. Ingram, and P. Maass, J. Non-Cryst. Solids 172-174, 1222 (1994).

[18] U. Schoo, C. Cramer, and H. Mehrer, Solid State Ionics 138, 105 (2000).

[19] Á. Imre, F. Berkemeier, H. Mehrer, Y. Gao, C. Cramer, and M. D. Ingram, J. Non-Cryst. Solids 354, 328 (2008).

[20] A. N. Cormack, J. Du, and T. R. Zeitler, Phys. Chem. Chem. Phys. 4, 3193 (2002).

[21] H. Mehrer, Á. W. Imre, and E. Tanguep-Nijokep, J. Phys: Conference Series 106, 012001 (2008).

[22] H. Lammert, M. Kunow, and A. Heuer, Phys. Rev. Lett. 90, 215901 (2003).

[23] M. Kodama and S. Kojima, Phys. Chem. Glasses 55, 1 (2014).

[24] J. C. Dyre, J. Appl. Phys. 64, 2456 (1988).

[25] 10.6084/m9.figshare.12651560.

[26] H. Jain, J. Non-Cryst. Solids 66, 517 (1984).

[27] Á. W. Imre, S. Voss, and H. Mehrer, Phys. Chem. Chem. Phys. 4, 3219 (2002).

[28] S. Murugavel and B. Roling, Phys. Rev. B 76, 180202(R) (2007).
[29] J. E. Shelby and S. C. Keeton, J. Appl. Phys. 45, 1458 (1974).

[30] R. Pecora, J. Chem. Phys. 40, 1604 (1964).

[31] R. Pecora, Dynamic Light Scattering-Applications of Photon Correlation Spectroscopy (Springer Science \& Business Media, Berlin, 2013).

[32] M. Sutton, S. G. J. Mochrie, T. Greytak, S. E. Nagler, L. E. Berman, G. A. Held, and G. B. Stephenson, Nature (London) 352, 608 (1991).

[33] G. Grübel, A. Madsen, and A. Robert, in Soft-Matter Characterization, edited by R. Borsali and R. Pecora (Springer Netherlands, Dordrecht, 2008), pp. 953-995.

[34] L. Cipelletti and E. R. Weeks, in Dynamical Heterogeneities in Glasses, Colloids and Granular Media, edited by L. Berthier, G. Biroli, J.-P. Bouchaud, L. Cipelletti, and W. van Saarlos (Oxford University Press, Oxford, 2011), pp. 110-151.

[35] H. Guo, G. Bourret, R. B. Lennox, M. Sutton, J. L. Harden, and R. L. Leheny, Phys. Rev. Lett. 109, 055901 (2012).

[36] M. Leitner, B. Sepiol, L.-M. Stadler, B. Pfau, and G. Vogl, Nat. Mater. 8, 717 (2009).

[37] M. Leitner, Studying Atomic Dynamics with Coherent X-rays (Springer, Berlin, 2012).

[38] M. Leitner and G. Vogl, J. Phys.: Condens. Matter 23, 254206 (2011).

[39] M. Stana, M. Leitner, M. Ross, and B. Sepiol, J. Phys.: Condens. Matter 25, 065401 (2013).

[40] M. Leitner, B. Sepiol, L.-M. Stadler, and B. Pfau, Phys. Rev. B 86, 064202 (2012).

[41] S. O. Hruszkewycz, M. Sutton, P. H. Fuoss, B. Adams, S. Rosenkranz, K. F. Ludwig, W. Roseker, D. Fritz, M. Cammarata, D. Zhu, S. Lee, H. Lemke, C. Gutt, A. Robert, G. Grübel, and G. B. Stephenson, Phys. Rev. Lett. 109, 185502 (2012).

[42] B. Ruta, Y. Chushkin, G. Monaco, L. Cipelletti, E. Pineda, P. Bruna, V. M. Giordano, and M. Gonzalez-Silveira, Phys. Rev. Lett. 109, 165701 (2012).

[43] M. Ross, M. Stana, M. Leitner, and B. Sepiol, New J. Phys. 16, 093042 (2014).

[44] V. M. Giordano and B. Ruta, Nat. Commun. 7, 10344 (2016).

[45] G. Pintori, G. Baldi, B. Ruta, and G. Monaco, Phys. Rev. B 99, 224206 (2019).

[46] B. Lengeler, Naturwissenschaften 88, 249 (2001).

[47] D. L. Abernathy, G. Grübel, S. Brauer, I. McNulty, G. B. Stephenson, S. G. J. Mochrie, A. R. Sandy, N. Mulders, and M. Sutton, J. Synchrotron Rad. 5, 37 (1998). 
[48] M. Sutton, in Neutron and X-ray Spectroscopy, edited by F. Hippert, E. Geissler, J. L. Hodeau, E. Leliévre-Berna, and J.-R. Regnard (Springer, Dordrecht, 2006), pp. 297-318.

[49] G. L. Squires, Introduction to the Theory of Thermal Neutron Scattering (Cambridge University Press, Cambridge, 1978).

[50] L. Van Hove, Phys. Rev. 95, 249 (1954).

[51] D. Lumma, L. B. Lurio, S. G. J. Mochrie, and M. Sutton, Rev. Sci. Instrum. 71, 3274 (2000).

[52] M. Sutton, C. R. Physique 9, 657 (2008).

[53] C. T. Chudley and R. J. Elliott, Proc. Phys. Soc. London 77, 353 (1961).

[54] R. Richert, J. Phys.: Condens. Matter 14, R703 (2002).

[55] 10.6084/m9.figshare.12651458.

[56] See Supplemental Material at http://link.aps.org/supplemental/ 10.1103/PhysRevResearch.2.043141 for a table of the model parameters fitted to the autocorrelation functions.

[57] S. V. Stolyar, L. V. Grishchenko, and G. A. Sycheva, Glass Phys. Chem. 32, 293 (2006).

[58] Á. W. Imre, H. Staesche, S. Voss, M. D. Ingram, K. Funke, and H. Mehrer, J. Phys. Chem. B 111, 5301 (2007).

[59] K. Holzweber, C. Tietz, T. M. Fritz, B. Sepiol, and M. Leitner, Phys. Rev. B 100, 214305 (2019).
[60] H. Kenmotsu, T. Hattori, S. Nishiyama, K. Fukushima, Y. Iwadate, M. Misawa, T. Fukunaga, T. Nakazawa, and K. Noda, J. Phys. Chem. Solids 60, 1461 (1999).

[61] C. A. Angell, Ann. Rev. Phys. Chem. 43, 693 (1992).

[62] G. D. Chryssikos, E. I. Kamitsos, and Y. D. Yiannopoulos, J. Non-Cryst. Solids 196, 244 (1996).

[63] G. D. Chryssikos, L. Liu, C. P. Varsamis, and E. I. Kamitsos, J. Non-Cryst. Solids 235-237, 761 (1998).

[64] B. Ruta, F. Zontone, Y. Chushkin, G. Baldi, G. Pintori, G. Monaco, B. Ruffle, and W. Kob, Sci. Rep. 7, 3962 (2017).

[65] L. Isserlis, Biometrika 12, 134 (1918).

[66] D. Sidebottom, R. Bergman, L. Börjesson, and L. M. Torell, Phys. Rev. Lett. 71, 2260 (1993).

[67] J. Horbach, W. Kob, and K. Binder, Phys. Rev. Lett. 88, 125502 (2002).

[68] M. Vogel, Phys. Rev. B 70, 094302 (2004).

[69] J. Habasaki and Y. Hiwatari, Phys. Rev. B 69, 144207 (2004).

[70] J. C. Dyre, J. Non-Cryst. Solids 324, 192 (2003).

[71] D. L. Sidebottom, P. F. Green, and R. K. Brow, J. Non-Cryst. Solids 222, 354 (1997).

[72] G. N. Greaves, J. Non-Cryst. Solids 71, 203 (1985).

[73] Cristos-Platon E. Varsamis, A. Vegiri, and E. I. Kamitsos, Phys. Rev. B 65, 104203 (2002). 